\title{
$D=4$ supergravity from the Maxwell-Weyl superalgebra
}

\author{
Salih Kibaroğlu ${ }^{1,2, a} \mathbb{C}$, Oktay Cebecioğlu $^{1, b}$ \\ ${ }^{1}$ Department of Physics, Kocaeli University, 41380 Kocaeli, Turkey \\ ${ }^{2}$ Institute for Theoretical Physics, University of Wrocław, Pl. Maksa Borna 9, Pl-50-204 Wrocław, Poland
}

Received: 27 December 2018 / Accepted: 24 October 2019 / Published online: 7 November 2019

(C) The Author(s) 2019

\begin{abstract}
We present the construction of the first-order $D=4, \mathcal{N}=1$ supergravity action by gauging the MaxwellWeyl superalgebra. The four-form lagrangian is constructed by using the curvatures of the algebra and the local scale invariance of the action is achieved through the introduction of a compensating scalar field. Finally, we find the generalized Einstein equation with a coordinate dependent cosmological term.
\end{abstract}

\section{Introduction}

The Maxwell symmetry appears if the Minkowski spacetime is filled with an additional background field [1,2]. In other words, this symmetry can be interpreted as a modification of the Poincaré symmetry which describes the empty Minkowski space-time, and the translation generators are no longer abelian but satisfy [3],

$\left[P_{a}, P_{b}\right]=i Z_{a b}$,

where the six additional antisymmetric generators $Z_{a b}$ transform as a second rank tensor under the action of Lorentz group. In early studies, this background field was associated with constant electromagnetic (EM) fields. Nowadays interpretation of the background field as well as this new algebra have opened up new directions. For example, this algebra was extensively studied to generalize Einstein's theory of gravity. In [4-7], the generalized cosmological constant and additional interaction terms were derived alternatively by extending the theory of gravity based on the Maxwell algebra. This fact may have a fundamental importance since the studies and observations on the cosmological constant, the dark energy and the cosmic microwave background indicate that there should be a background field filling our space-time. We also know that there is a close connection between cos-

\footnotetext{
a e-mail: salihkibaroglu@gmail.com

b e-mail: ocebecioglu@kocaeli.edu.tr
}

mological constant and dark energy [8,9], so the Maxwell symmetries may provide a powerful geometrical framework for these significant topics.

The supersymmetric extension of the Maxwell algebra was presented in [10] and it describes the geometry of $D=4$, $\mathcal{N}=1$ superspace with a constant abelian supersymmetric gauge field background. This modification of superspace is known as the superMaxwell space. Contrary to the conventional superalgebras, this superalgebra contains two Majorana spinor generators $Q_{\alpha}$ and $\Sigma_{\alpha}$. This superalgebra can be considered as a generalization of the D'Auria-Fré superalgebra [11] and the Green algebra [12] which have additional fermionic generators. Besides, the Maxwell superalgebras were also obtained in $[13,14]$ by using algebraic expansion methods $[15,16]$. In [17], the authors derived the first order $D=4, \mathcal{N}=1$ pure supergravity lagrangian fourform by using the curvatures of the Maxwell superalgebra. Subsequently, the generalized supersymmetric cosmological constant was constructed based on the Maxwell superalgebras in $\mathcal{N}=1$ case $[18,19]$. Beyond the case of $\mathcal{N}=1$, the $\mathcal{N}$-extended Maxwell superalgebras were considered in $[13,14,20,21]$. Recent developments and some interesting studies about the Maxwell (super)algebras can be found in [22-35].

The Weyl enlargement of the Maxwell algebra, which named as the Maxwell-Weyl algebra $\mathcal{M W}$, and its supersymmetric extension $s \mathcal{M W}$ were firstly presented in [24]. In our earlier work, we constructed a gauge theory of gravity based on $\mathcal{M W}$ and obtained the generalized Einstein equation with cosmological constant [7]. The main purpose of this letter is to establish a gauge theory of gravity based on $s \mathcal{M W}$ and to construct the first-order $D=4, \mathcal{N}=1$ supergravity action.

The paper organized as follows. In Sect. 2, we give a brief summary of the $\mathcal{M W}$ algebra and its gravity action. In Sect. 3, we introduce $s \mathcal{M W}$ algebra and obtain transformation of the gauge fields, the curvatures and the Bianchi identities of the algebra. In Sect. 4, the supergravity action is con- 
structed by using the curvatures of $s \mathcal{M W}$ algebra together with an additional compensating scalar field. In Sect. 5, we conclude the paper with some comments. In the last section, our notations and conventions are given.

\section{Maxwell-Weyl algebra and the gravity action}

In this section, we briefly give the results of our previous study [7]. The Maxwell-Weyl algebra $\mathcal{M W}$ can be considered as Weyl enlargement of the Maxwell algebra with a dilatation generator [24]. The non-zero commutation relations of $\mathcal{M W}$ algebra are given by,

$$
\begin{aligned}
{\left[M_{a b}, M_{c d}\right] } & =i\left(\eta_{a d} M_{b c}+\eta_{b c} M_{a d}-\eta_{a c} M_{b d}-\eta_{b d} M_{a c}\right), \\
{\left[P_{a}, P_{b}\right] } & =i Z_{a b}, \\
{\left[M_{a b}, P_{c}\right] } & =i\left(\eta_{b c} P_{a}-\eta_{a c} P_{b}\right), \\
{\left[M_{a b}, Z_{c d}\right] } & =i\left(\eta_{a d} Z_{b c}+\eta_{b c} Z_{a d}-\eta_{a c} Z_{b d}-\eta_{b d} Z_{a c}\right), \\
{\left[P_{a}, D\right] } & =i P_{a}, \\
{\left[Z_{a b}, D\right] } & =2 i Z_{a b},
\end{aligned}
$$

where $\eta_{a b}$ is the Minkowski metric which has diag $\left(\eta_{a b}\right)=$ $(+,-,-,-)$ and the indices $a, b, \ldots=0, \ldots, 3$. In addition to the Weyl algebra, this algebra contains six new additional tensorial generators $Z_{a b}$.

Let us start to construct the gauge theory of gravity based on $\mathcal{M W}$ algebra. We first introduce the following one-form gauge field with,

$\mathcal{A}=e^{a} P_{a}+B^{a b} Z_{a b}+\chi D-\frac{1}{2} \omega^{a b} M_{a b}$,

where the coefficient fields $e^{a}(x), B^{a b}(x), \chi(x)$ and $\omega^{a b}(x)$ are the one-form gauge fields for the corresponding generators, respectively. Using the structure equation $\mathcal{F}=$ $d \mathcal{A}+\frac{i}{2}[\mathcal{A}, \mathcal{A}]$ and defining the curvatures as $\mathcal{F}=F^{a} P_{a}+$ $F^{a b} Z_{a b}+f D-\frac{1}{2} R^{a b} M_{a b}$, we find the associated two-form curvatures as,

$$
\begin{aligned}
F^{a} & =d e^{a}+\omega_{b}^{a} \wedge e^{b}+\chi \wedge e^{a}, \\
F^{a b} & =d B^{a b}+\omega^{[a}{ }_{c} \wedge B^{c \mid b]}+2 \chi \wedge B^{a b}-\frac{1}{2} e^{a} \wedge e^{b}, \\
f & =d \chi, \\
R^{a b} & =d \omega^{a b}+\omega^{a}{ }_{c} \wedge \omega^{c b} .
\end{aligned}
$$

The infinitesimal gauge transformation of the curvatures can be found by introducing the Lie algebra valued parameters,

$$
\begin{aligned}
\zeta(x)= & y^{a}(x) P_{a}+\varphi^{a b}(x) Z_{a b} \\
& +\rho(x) D-\frac{1}{2} \tau^{a b}(x) M_{a b},
\end{aligned}
$$

with the help of the equation $\delta \mathcal{F}=i[\zeta, \mathcal{F}]$. Here, $y^{a}(x)$, $\varphi^{a b}(x), \rho(x)$ and $\tau^{a b}(x)$ are the space-time translations, translation in tensorial space, dilatation parameter, and the
Lorentz transformation parameters respectively. Thus, the transformations of the curvatures under $\mathcal{M W}$ algebra are found as follows,

$$
\begin{aligned}
\delta F^{a} & =\omega_{b}^{a} F^{b}+\rho F^{a}-R_{b}^{a} y^{b}-f y^{a}, \\
\delta F^{a b} & =\omega^{[a}{ }_{c}^{c \mid b]}+2 \rho F^{a b}+\frac{1}{2} F^{[a} y^{b]}-R_{c}^{[a} \varphi^{c \mid b]}-\frac{1}{2} f \varphi^{a b}, \\
\delta f & =0, \\
\delta R^{a b} & =\omega^{[a}{ }_{c}^{c \mid b]} .
\end{aligned}
$$

Taking the covariant derivative of given curvatures, the corresponding Bianchi identities can be found as follows

$$
\begin{aligned}
\mathcal{D} F^{a} & =R^{a}{ }_{b} \wedge e^{b}+f \wedge e^{a}, \\
\mathcal{D} F^{a b} & =R^{[a}{ }_{c} \wedge B^{c \mid b]}+2 f \wedge B^{a b}-\frac{1}{2} F^{[a} \wedge e^{b]}, \\
\mathcal{D} f & =0 \\
\mathcal{D} R^{a b} & =0
\end{aligned}
$$

where $\mathcal{D} \Phi=[d+\omega+w(\Phi) \chi] \Phi$ is the Lorentz-Weyl covariant derivative and $w$ being the Weyl weight of the corresponding field.

Construction of a lagrangian based on $\mathcal{M W}$ algebra is slightly different from the ordinary Maxwell algebra because $\mathcal{M W}$ algebra contains the scale transformations which act on fields as

$$
\Phi^{\prime}(x)=e^{w \rho(x)} \Phi(x)
$$

where $\rho(x)$ is local scale parameter and $w$ is the Weyl weight of scalar field $\Phi(x)$ (for more detail about the Weyl transformation see [36-41]). In our context, transformation of the metric tensor with respect to the Weyl transformation is $g_{\mu \nu}^{\prime}=e^{2 \rho(x)} g_{\mu \nu}$. Thus, the variation of metric tensor satisfies

$\delta g_{\mu \nu}(x)=2 \rho(x) g_{\mu \nu}(x)$

under infinitesimal gauge transformations, where $\mu, v=$ $0, \ldots, 3$ are space-time indices. This means that the Weyl weight of the metric tensor is $w\left(g_{\mu \nu}\right)=2, w\left(g^{\mu \nu}\right)=-2$ and $w(\sqrt{-g})=4$. For this reason, the well-known action $S=\int d^{4} x \sqrt{-g} R$ is not invariant under the local scale transformations. To overcome this difficulty, Weyl used quadratic terms in the action to construct a consistent theory [42]. Furthermore, Brans and Dicke constructed a lagrangian with the combination of the scalar curvature $R$ and a new compensating scalar field $\phi(x)$ which have a Weyl weight [43]. Later, Dirac used the scalar field $\phi(x)$ with having $w(\phi)=-1$ and constructed the action by using $\phi^{2} R$ rather than $R$ [44] (for more information see [45,46]).

From these theoretical backgrounds, in accordance with Dirac's convention, we introduced a compensating scalar field $\phi(x)$ which transforms as $\delta \phi=-\rho \phi$ in order to make the Einstein-Hilbert action to be local scale invariant (more detail see $[7,44])$. Now, if we define a shifted curvature as 
$\mathcal{J}^{a b}=R^{a b}+2 \gamma \phi^{2} F^{a b}$, we can write the following action having Weyl weight zero,

$S=\int \frac{1}{2 \kappa \gamma} \mathcal{J} \wedge^{*} \mathcal{J}+\frac{1}{4} f \wedge^{*} f-\frac{1}{2} \mathcal{D} \phi \wedge^{*} \mathcal{D} \phi+\frac{\lambda}{4} \phi^{4 *} 1$,

where $\kappa, \gamma$ and $\lambda$ are constants and $*$ represents the Hodge duality operator.

\section{Gauging the maxwell-weyl superalgebra}

In this section, we consider gauge theory of the $s \mathcal{M W}$ algebra. The Weyl enlargement of the Maxwell superalgebra was first proposed in [24] with a different notation. In our convention, we can write the $s \mathcal{M W}$ algebra in addition to Eq. (2) as

$$
\begin{aligned}
\left\{Q_{\alpha}, Q_{\beta}\right\} & =2\left(C \gamma^{c}\right)_{\alpha \beta} P_{c}, \\
{\left[P_{a}, Q_{\beta}\right] } & =-i\left(\gamma_{a} \Sigma\right)_{\beta}, \\
\left\{Q_{\alpha}, \Sigma_{\beta}\right\} & =-\frac{i}{2}\left(C \sigma^{c d}\right)_{\alpha \beta} Z_{c d}-i\left(C \gamma_{5}\right)_{\alpha \beta} B \\
{\left[M_{a b}, Q_{\rho}\right] } & =\frac{1}{2}\left(\sigma_{a b} Q\right)_{\rho}, \quad\left[M_{a b}, \Sigma_{\rho}\right]=\frac{1}{2}\left(\sigma_{a b} \Sigma\right)_{\rho}, \\
{[D, B] } & =-2 i B, \\
{\left[D, Q_{\alpha}\right] } & =-\frac{i}{2} Q_{\alpha}, \quad\left[D, \Sigma_{\alpha}\right]=-\frac{3 i}{2} \Sigma_{\alpha} .
\end{aligned}
$$

Here $Q_{\alpha}$ are the standard supersymmetric fermionic charges where the spinor indices $\alpha, \beta, \ldots=1, \ldots, 4$. To close the commutator $[P, Q]$ satisfying at the same time the Jacobi identities $(P, Q, Q)$, we need to introduce an additional fermionic generator $\Sigma_{\alpha}$ as in $[10,24]$. In first glance, the presence of an extra fermionic charge in the theory means a second gravitino, but it is not so. As we will see later, the sigma generator does not contribute the spacetime coordinates. Only contribution from the fermionic charges to the spacetime coordinates comes from $Q_{\alpha}$ supercharges. In $\mathcal{N}=1$ case, the motivation for introducing extra fermionic generators can be found in [11,12] and they can also be obtained by taking into account of the algebraic expansion methods $[15,16]$. The central charge $B$ describes the off-shell extension of $U$ (1) field strength multiplet in $D=4$. If we remove $B$, which is mathematically possible, we get the minimal Maxwell-Weyl superalgebra (for more detail see [24]). For the closure of this superalgebra, we need to use the cyclic identities given as $\left(C \gamma_{e}\right)_{(\alpha \beta}\left(C \gamma^{e}\right)_{\rho \sigma)}=0$. All spinors given in this paper are characterized by Majorana spinors and satisfying the generic relation $\bar{Q}=Q^{T} C$ where $C$ is the charge conjugation matrix.

To gauge this algebra, we start by introducing a one-form connection $\mathcal{A}(x)$ as,

$$
\begin{aligned}
\mathcal{A}(x)= & e^{a} P_{a}+B^{a b} Z_{a b}+\chi D \\
& -\frac{1}{2} \omega^{a b} M_{a b}+\psi^{\alpha} Q_{\alpha}+\xi^{\alpha} \Sigma_{\alpha}+A B .
\end{aligned}
$$

Comparing with Eq. (3), the last expression contains three additional gauge fields $\psi^{\alpha}(x), \xi^{\alpha}(x)$, and $A(x)$ which correspond to the gravitino field, the additional fermionic gauge field and gauge field of central charge $B$, respectively. The variations of these fields under infinitesimal gauge transformation of $s \mathcal{M W}$ can be obtained by $\delta \mathcal{A}=-d \zeta+i[\zeta, \mathcal{A}]$, where $\zeta(x)$ is the Lie algebra valued auxiliary field defined as,

$$
\begin{aligned}
\zeta(x)= & y^{a} P_{a}+\varphi^{a b} Z_{a b}+\rho D \\
& -\frac{1}{2} \tau^{a b} M_{a b}+\varepsilon^{\alpha} Q_{\alpha}+v^{\alpha} \Sigma_{\alpha}+r B .
\end{aligned}
$$

In addition to Eq. (5), $\varepsilon^{\alpha}(x), v^{\alpha}(x)$ and $r(x)$ represent the parameters of $Q_{\alpha}, \Sigma_{\alpha}$ and $B$, respectively. From these definitions, one can find transformation of the gauge fields as,

$$
\begin{aligned}
\delta e^{a}= & -\mathcal{D} y^{a}+\tau_{b}^{a} e^{b}+\rho e^{a}-2 i \bar{\varepsilon} \gamma^{a} \psi, \\
\delta B^{a b}= & -\mathcal{D} \varphi^{a b}+\tau^{[a}{ }_{c}^{c} B^{c \mid b]}+2 \rho B^{a b}+\frac{1}{2} e^{[a} y^{b]} \\
& -\frac{1}{2} \bar{\varepsilon} \sigma^{a b} \xi-\frac{1}{2} \bar{v} \sigma^{a b} \psi, \\
\delta \chi= & -d \rho, \\
\delta \omega^{a b}= & -\mathcal{D} \tau^{a b}, \\
\delta \psi^{\alpha}= & -\mathcal{D} \varepsilon^{\alpha}-\frac{i}{4} \tau^{a b}\left(\sigma_{a b} \psi\right)^{\alpha}+\frac{1}{2} \rho \psi^{\alpha}, \\
\delta \xi^{\alpha}= & -\mathcal{D} v^{\alpha}-\frac{i}{4} \tau^{a b}\left(\sigma_{a b} \xi\right)^{\alpha}+\frac{3}{2} \rho \xi^{\alpha} \\
& +y^{c}\left(\gamma_{c} \psi\right)^{\alpha}-e^{c}\left(\gamma_{c} \varepsilon\right)^{\alpha}, \\
\delta A= & -\mathcal{D} r-2 \rho A-\bar{\varepsilon} \gamma_{5} \xi+\bar{v} \gamma_{5} \psi,
\end{aligned}
$$

where $\mathcal{D}$ is the Lorentz-Weyl covariant derivative which was given in Sect. 2. To find the associated two-form curvatures of corresponding superalgebra, we use the well-known relation $\mathcal{F}=d \mathcal{A}+\frac{i}{2}[\mathcal{A}, \mathcal{A}]$, where $\mathcal{F}$ represents the curvatures and it is given in the following form,

$$
\begin{aligned}
\mathcal{F}= & F^{a} P_{a}+F^{a b} Z_{a b}+f D \\
& -\frac{1}{2} R^{a b} M_{a b}+\Psi^{\alpha} Q_{\alpha}+\Xi^{\alpha} \Sigma_{\alpha}+K B .
\end{aligned}
$$

Therefore, the explicit form of the curvatures can be found as follows,

$$
\begin{aligned}
F^{a}= & d e^{a}+\omega^{a}{ }_{b} \wedge e^{b}+\chi \wedge e^{a}-i\left(\bar{\psi} \wedge \gamma^{a} \psi\right), \\
F^{a b}= & d B^{a b}+\omega^{[a}{ }_{c} \wedge B^{c \mid b]}+2 \chi \wedge B^{a b} \\
& -\frac{1}{2} e^{a} \wedge e^{b}-\frac{1}{2}\left(\bar{\psi} \wedge \sigma^{a b} \xi\right), \\
f= & d \chi \\
R^{a b}= & d \omega^{a b}+\omega^{a}{ }_{c} \wedge \omega^{c b}, \\
\Psi^{\alpha}= & d \psi^{\alpha}-\frac{i}{4} \omega^{a b} \wedge\left(\sigma_{a b} \psi\right)^{\alpha}+\frac{1}{2} \chi \wedge \psi^{\alpha},
\end{aligned}
$$




$$
\begin{aligned}
\Xi^{\alpha} & =d \xi^{\alpha}-\frac{i}{4} \omega^{a b} \wedge\left(\sigma_{a b} \xi\right)^{\alpha}+\frac{3}{2} \chi \wedge \xi^{\alpha}+e^{c} \wedge\left(\gamma_{c} \psi\right)^{\alpha} \\
K & =d A+2 \chi \wedge A-\left(\bar{\psi} \gamma_{5} \xi\right)
\end{aligned}
$$

The curvatures have the following length dimensions: $\left[F^{a}\right]=$ $L,\left[F^{a b}\right]=L^{2},[f]=L^{0},\left[R^{a b}\right]=L^{0},\left[\Psi^{\alpha}\right]=L^{1 / 2}$, $\left[\Xi^{\alpha}\right]=L^{3 / 2}$ and $[K]=L^{2}$. Here, if we take $\mathcal{F}=0$, we find the Maurer-Cartan equations for $s \mathcal{M W}$. The infinitesimal gauge transformation of the curvatures under $s \mathcal{M W}$ group can be found by using the relation $\delta \mathcal{F}=i[\zeta, \mathcal{F}]$ as,

$$
\begin{aligned}
\delta F^{a}= & \omega^{a}{ }_{b} F^{b}+\rho F^{a}-R^{a}{ }_{b} y^{b}-f y^{a}-2 i \bar{\varepsilon} \gamma^{a} \Psi, \\
\delta F^{a b}= & \omega^{[a}{ }_{c} F^{c \mid b]}+2 \rho F^{a b}+\frac{1}{2} F^{[a} y^{b]} \\
& -R^{[a}{ }_{c} \varphi^{c \mid b]}-\frac{1}{2} f \varphi^{a b} \\
& -\frac{1}{2} \bar{\varepsilon} \sigma^{a b} \Xi-\frac{1}{2} \bar{v} \sigma^{a b} \Psi, \\
\delta f= & 0, \\
\delta R^{a b}= & \omega^{[a}{ }_{c} R^{c \mid b]}, \\
\delta \Psi^{\alpha}= & -\frac{i}{4} \tau^{a b}\left(\sigma_{a b} \Psi\right)^{\alpha}+\frac{1}{2} \rho \Psi^{\alpha} \\
& +\frac{i}{4} R^{a b}\left(\sigma_{a b} \varepsilon\right)^{\alpha}-\frac{1}{2} \varepsilon^{\alpha} f, \\
\delta \Xi^{\alpha}= & -\frac{i}{4} \tau^{a b}\left(\sigma_{a b} \Xi\right)^{\alpha}+\frac{3}{2} \rho \Xi^{\alpha}+\frac{i}{4} R^{a b}\left(\sigma_{a b} v\right)^{\alpha} \\
& -\frac{3}{2} v^{\alpha} f+y^{c}\left(\gamma_{c} \Psi\right)^{\alpha}+F^{c}\left(\gamma_{c} \varepsilon\right)^{\alpha}, \\
\delta K= & 2 \rho K-2 r f-\bar{k} \gamma_{5} \Xi+\bar{v} \gamma_{5} \Psi .
\end{aligned}
$$

Finally, the Bianchi identities of corresponding curvatures can be obtained as follows,

$$
\begin{aligned}
\mathcal{D} F^{a}= & R^{a}{ }_{b} \wedge e^{b}+f \wedge e^{a}+2 i \bar{\psi} \gamma^{a} \Psi, \\
\mathcal{D} F^{a b}= & R^{[a}{ }_{c} \wedge B^{c \mid b]}+2 f \wedge B^{a b} \\
& -\frac{1}{2} F^{[a} \wedge e^{b]}-\frac{1}{2} \bar{\Psi} \wedge \sigma^{a b} \xi \\
& +\frac{1}{2} \bar{\psi} \wedge \sigma^{a b} \Xi+\frac{1}{2} e^{c} \wedge\left(\bar{\psi} \wedge \sigma^{a b} \gamma_{c} \psi\right), \\
\mathcal{D} f= & 0, \\
\mathcal{D} R^{a b}= & 0, \\
\mathcal{D} \Psi^{\alpha}= & -\frac{i}{4} R^{a b} \wedge\left(\sigma_{a b} \psi\right)^{\alpha}+\frac{1}{2} f \wedge \psi^{\alpha}, \\
\mathcal{D} \Xi^{\alpha}= & -\frac{i}{4} R^{a b} \wedge\left(\sigma_{a b} \xi\right)^{\alpha} \\
& +\frac{3}{2} f \wedge \xi^{\alpha}+F^{c} \wedge\left(\gamma_{c} \psi\right)^{\alpha}-e^{c} \wedge\left(\gamma_{c} \Psi\right)^{\alpha}, \\
\mathcal{D} K= & 2 f \wedge A-\bar{\Psi} \wedge \gamma_{5} \xi+\bar{\psi} \wedge \gamma_{5} \Xi .
\end{aligned}
$$

\section{Constructing $D=4, \mathcal{N}=1$ supergravity action}

To construct scale invariant theory of gravity, as we mentioned in Sect. 2, we will use the method of the Brans-
Dicke theory of gravitation [43] with the Dirac formalism [44] which contains a scalar compensating field $\phi(x)$ with $w(\phi)=-1$. Following a similar method to [7,17,47], we will establish a lagrangian by using the curvature bilinear constructed out of $s \mathcal{M W}$ algebra together with the additional scalar field (more details on supergravity including the local scale transformation can be found in [48-50]).

In $s \mathcal{M W}$ framework, the metric tensor shows the same characteristics with Eq. (9) so it has $w\left(g_{\mu \nu}\right)=2$. According to Eq. (14), the Weyl weights of the gauge fields are defined as $w\left(e_{\mu}^{a}\right)=1, w\left(B_{\mu}^{a b}\right)=2, w\left(\chi_{\mu}\right)=0, w\left(\omega_{\mu}^{a b}\right)=0$, $w\left(\psi_{\mu}^{\alpha}\right)=1 / 2, w\left(\xi_{\mu}^{\alpha}\right)=3 / 2$, and $w\left(A_{\mu}\right)=2$. Similarly, the Weyl weights of the curvatures can be found from Eq. (17). Finally, we can write a free gravitational four-form lagrangian density as follows,

$L_{f}=\frac{\phi^{2}}{2 \kappa}\left(\varepsilon_{a b c d} R^{a b} \wedge F^{c d}+4 \bar{\Xi} \wedge \gamma_{5} \Psi-2 f \wedge K\right)$,

where $\kappa$ is a constant. In addition, one can use the following lagrangian for vacuum,

$L_{0}=\frac{1}{4} f \wedge^{*} f-\frac{1}{2} \mathcal{D} \phi \wedge^{*} \mathcal{D} \phi+\frac{\lambda}{4} \phi^{4 *} 1$,

where $\lambda$ is another constant, the first term represents a Maxwell like kinetic term, the second corresponds to the kinetic term for the compensating scalar field and the last one is a self-interaction term for the scalar field. Finally, the total action can be written as follows,

$S=\int L_{f}+L_{0}$

In order to simplify the total action, let us expand the free part $L_{f}$ as follows,

$$
\begin{aligned}
S_{f}= & \frac{1}{2 \kappa} \int \phi^{2} \varepsilon_{a b c d} R^{a b} \wedge \mathcal{D} B^{c d}-\frac{1}{2} \phi^{2} \varepsilon_{a b c d} R^{a b} \wedge e^{c} \wedge e^{d} \\
& -\frac{1}{2} \phi^{2} \varepsilon_{a b c d} R^{a b} \wedge\left(\bar{\xi} \wedge \sigma^{c d} \psi\right)+4 \phi^{2} \mathcal{D} \bar{\xi} \wedge \gamma_{5} \Psi \\
& +4 \phi^{2} \bar{\psi} \wedge e_{a} \gamma^{a} \gamma_{5} \wedge \Psi-2 \phi^{2} f \wedge\left(\mathcal{D} A-\bar{\psi} \wedge \gamma_{5} \xi\right) \\
= & \frac{1}{2 \kappa} \int-\frac{1}{2} \phi^{2}\left(\varepsilon_{a b c d} R^{a b} \wedge e^{c} \wedge e^{d}-8 \bar{\psi} \wedge e_{a} \wedge \gamma^{a} \gamma_{5} \mathcal{D} \psi\right) \\
& +\mathcal{D}\left(\phi^{2} \varepsilon_{a b c d} R^{a b} \wedge B^{c d}-2 \phi^{2} f \wedge A\right) \\
& -\mathcal{D} \phi^{2}\left(\varepsilon_{a b c d} R^{a b} \wedge B^{c d}-2 f \wedge A\right) \\
& -\frac{1}{2} \phi^{2} \varepsilon_{a b c d} R^{a b} \wedge\left(\bar{\xi} \wedge \sigma^{c d} \psi\right) \\
& +4 \phi^{2} \mathcal{D} \bar{\xi} \wedge \gamma_{5} \Psi+2 \phi^{2} f \wedge\left(\bar{\psi} \wedge \gamma_{5} \xi\right)
\end{aligned}
$$

where the term $4 \phi^{2} \mathcal{D} \bar{\xi} \gamma_{5} \wedge \Psi$ can be expanded by using the Bianchi identity $\mathcal{D} \Psi$ given in Eq. (19) together with the relation $\gamma_{5} \sigma_{a b}=\frac{i}{2} \varepsilon_{a b c d} \sigma^{c d}$ as follows,

$$
\begin{aligned}
& 4 \phi^{2} \mathcal{D} \bar{\xi} \wedge \gamma_{5} \Psi \\
& =\mathcal{D}\left(4 \phi^{2} \bar{\xi} \wedge \gamma_{5} \Psi\right)+4 \mathcal{D} \phi^{2}\left(\bar{\xi} \wedge \gamma_{5} \Psi\right)
\end{aligned}
$$




$$
+\frac{1}{2} \phi^{2} \varepsilon_{a b c d} R^{a b} \wedge\left(\bar{\xi} \wedge \sigma^{c d} \psi\right)-2 \phi^{2} f \wedge\left(\bar{\psi} \wedge \delta_{5} \xi\right) .
$$

Substituting Eq. (24) in Eq. (23), the free lagrangian density is reduced to the following form,

$$
\begin{aligned}
S_{f}= & \frac{1}{2 \kappa} \int-\frac{1}{2} \phi^{2}\left(\varepsilon_{a b c d} R^{a b} \wedge e^{c} \wedge e^{d}-8 \bar{\psi} \wedge e_{a} \wedge \gamma^{a} \gamma_{5} \mathcal{D} \psi\right) \\
& -\mathcal{D} \phi^{2}\left(\varepsilon_{a b c d} R^{a b} \wedge B^{c d}-4 \bar{\xi} \wedge \gamma_{5} \Psi-2 f \wedge A\right) \\
& +\mathcal{D}\left(\phi^{2} \varepsilon_{a b c d} R^{a b} \wedge B^{c d}+4 \phi^{2} \bar{\xi} \wedge \gamma_{5} \Psi-2 \phi^{2} f \wedge A\right),
\end{aligned}
$$

where the first part of the resulting action represents the Rarita-Schwinger term including the compensating scalar field, the second is an extra term and the third one is total derivative. It can be easily found that the total action in Eq. (22) is clearly invariant under the local Lorentz and scale gauge transformations. Let us now check the invariance of the action in Eq. (25) under the local supersymmetry transformation. To do this, take the first term in Eq. (25) and name it as $S_{f}^{1}$. Using Eq. (14), the variation of $S_{f}^{1}$ becomes,

$$
\begin{aligned}
\delta_{\text {susy }} & S_{f}^{1} \\
= & -\frac{1}{4 \kappa} \int-4 \phi^{2} i \epsilon_{a b c d} R^{a b} \wedge \bar{\varepsilon} \gamma^{c} \psi \wedge e^{d} \\
& -8 \phi^{2} \mathcal{D} \bar{\varepsilon} \wedge \gamma_{5} \gamma_{a} \mathcal{D} \psi \wedge e^{a} \\
& -8 \phi^{2} \bar{\psi} \wedge \gamma_{5} \gamma_{a} \mathcal{D}^{2} \varepsilon \wedge e^{a} \\
& -16 i \phi^{2} \bar{\psi} \wedge \gamma_{5} \gamma_{a} \mathcal{D} \psi \wedge \bar{\varepsilon} \gamma^{a} \psi .
\end{aligned}
$$

With the help of partial integration and the super-torsion $F^{a}$, the second term in Eq. (26) turns into,

$$
\begin{aligned}
- & 8 \phi^{2} \mathcal{D} \bar{\varepsilon} \wedge \gamma_{5} \gamma_{a} \mathcal{D} \psi \wedge e^{a} \\
= & \mathcal{D}\left(8 \phi^{2} \bar{\varepsilon} \gamma_{5} \gamma_{a} \mathcal{D} \psi \wedge e^{a}\right)+8 \mathcal{D} \phi^{2} \wedge \bar{\varepsilon} \gamma_{5} \gamma_{a} \mathcal{D} \psi \wedge e^{a} \\
& +8 \phi^{2} \bar{\varepsilon} \gamma_{5} \gamma_{a} \mathcal{D}^{2} \psi \wedge e^{a} \\
& +8 \phi^{2} \bar{\varepsilon} \gamma_{5} \gamma_{a} \mathcal{D} \psi \wedge F^{a}+8 \phi^{2} i \bar{\varepsilon} \gamma_{5} \gamma_{a} \mathcal{D} \psi \wedge \bar{\psi} \wedge \gamma^{a} \psi .
\end{aligned}
$$

Ignoring the total derivative and substituting the last result into Eq. (26), we get,

$$
\begin{aligned}
\delta_{\text {susy }} & S_{f}^{1} \\
= & -\frac{1}{4 \kappa} \int-4 i \phi^{2} \epsilon_{a b c d} R^{a b} \wedge \bar{\varepsilon} \gamma^{c} \psi \wedge e^{d} \\
& +8 \phi^{2} \bar{\varepsilon} \wedge \gamma_{5} \gamma_{a} \mathcal{D}^{2} \psi \wedge e^{a} \\
& +8 \phi^{2} \bar{\varepsilon} \wedge \gamma_{5} \gamma_{a} \mathcal{D} \psi \wedge F^{a} \\
& +8 i \phi^{2} \bar{\varepsilon} \wedge \gamma_{5} \gamma_{a} \mathcal{D} \psi \wedge \bar{\psi} \wedge \gamma^{a} \psi \\
& -8 \phi^{2} \bar{\psi} \wedge \gamma_{5} \gamma_{a} \mathcal{D}^{2} \varepsilon \wedge e^{a} \\
& -16 \phi^{2} \bar{\psi} \wedge \gamma_{5} \gamma_{a} \mathcal{D} \psi \wedge \bar{\varepsilon} \gamma^{a} \psi \\
& +8 \mathcal{D} \phi^{2} \wedge \bar{\varepsilon} \wedge \gamma_{5} \gamma_{a} \mathcal{D} \psi \wedge e^{a} .
\end{aligned}
$$

Considering the second and fifth terms in Eq. (28), one obtains,

$$
\begin{aligned}
& 8 \phi^{2} \bar{\varepsilon} \gamma_{5} \gamma_{a} \mathcal{D}^{2} \psi \wedge e^{a}-8 \phi^{2} \bar{\psi} \wedge \gamma_{5} \gamma_{a} \mathcal{D}^{2} \varepsilon \wedge e^{a} \\
& \quad=4 i \phi^{2} \epsilon_{a b c d} R^{a b} \wedge \bar{\varepsilon} \gamma^{c} \psi \wedge e^{d} .
\end{aligned}
$$

Here, $\gamma_{5} \gamma_{a} \gamma_{b c}=i \gamma_{5} \eta_{a[b} \gamma_{c]}+\epsilon_{a b c d} \gamma^{d}$ and $\mathcal{D}^{2} \Upsilon=$ $-\frac{i}{4} R^{a b} \gamma_{a b} \Upsilon+w(\Upsilon) f \Upsilon$ have been used where $\Upsilon$ is a spinor field. Therefore, the last result and the first term in Eq.(28) cancel each other, so we get,

$$
\begin{aligned}
\delta_{\text {susy }} S_{f}^{1} \\
=-\frac{1}{4 \kappa} \int 8 \bar{\varepsilon} \gamma_{5} \gamma_{a} \mathcal{D} \psi \wedge F^{a}+8 \mathcal{D} \phi^{2} \wedge \bar{\varepsilon} \gamma_{5} \gamma_{a} \mathcal{D} \psi \wedge e^{a} \\
\quad+8 i \phi^{2}\left(\bar{\varepsilon} \gamma_{5} \gamma_{a} \mathcal{D} \psi \wedge \bar{\psi} \wedge \gamma^{a} \psi+2 \bar{\varepsilon} \gamma^{a} \psi \wedge \bar{\psi} \wedge \gamma_{5} \gamma_{a} \mathcal{D} \psi\right) .
\end{aligned}
$$

Using the relations $\psi \wedge \bar{\psi}=\frac{1}{2}\left(\bar{\psi} \wedge \gamma^{a} \psi\right)$ $\gamma_{a}-\frac{1}{8}\left(\bar{\psi} \wedge \gamma^{a b} \psi\right) \gamma_{a b}, \gamma_{a} \gamma^{b c} \gamma^{a}=0$ and $\gamma^{a} \gamma^{b} \gamma_{5} \gamma_{a}=$ $2 \gamma^{b} \gamma_{5}$, the fourth term becomes,

$2 \bar{\varepsilon} \gamma^{a} \psi \wedge \bar{\psi} \wedge \gamma_{5} \gamma_{a} \mathcal{D} \psi=-\bar{\varepsilon} \gamma_{5} \gamma_{a} \mathcal{D} \psi \wedge\left(\bar{\psi} \wedge \gamma^{a} \psi\right)$,

then substituting the last equation into Eq. (30), the variation reduces to

$\delta_{\text {susy }} S_{f}^{1}=-\frac{1}{4 \kappa} \int 8 \bar{\varepsilon} \gamma_{5} \gamma_{a} \mathcal{D} \psi \wedge F^{a}-8 \mathcal{D} \phi^{2} \wedge e_{a} \wedge \bar{\varepsilon} \gamma^{a} \gamma_{5} \mathcal{D} \psi$.

Assuming the constraints as $F^{a}=0$ and $\mathcal{D} \phi=0, \delta_{\text {susy }} S_{f}^{1}$ becomes zero. Under these constraints, Eq. (25) finally reduces to pure scale-invariant supergravity action with a boundary term,

$$
\begin{aligned}
S_{f}= & \frac{1}{2 \kappa} \int-\frac{1}{2} \phi^{2}\left(\varepsilon_{a b c d} R^{a b} \wedge e^{c} \wedge e^{d}-8 \bar{\psi} \wedge e_{a} \wedge \gamma^{a} \gamma_{5} \mathcal{D} \psi\right) \\
& +\mathcal{D}\left(\phi^{2} \varepsilon_{a b c d} R^{a b} \wedge B^{c d}+4 \phi^{2} \bar{\xi} \wedge \gamma_{5} \Psi-2 \phi^{2} f \wedge A\right) .
\end{aligned}
$$

Therefore, the action $S_{f}$ leave invariant under the local supersymmetry transformations in terms of given conditions. The additional fields $B^{a b}(x), \xi^{\alpha}(x)$ and $A(x)$ appeared only in the boundary term so we can say that they do not affect the dynamics of the action. This result also shows that $s \mathcal{M W}$ algebra provides an alternative way to construct the supergravity lagrangian including the local scale invariance. Finally, ignoring the boundary term and applying the condition to $L_{0}$, the total action in Eq. (22) takes the following form,

$$
\begin{aligned}
S= & \int-\frac{1}{4 \kappa} \phi^{2}\left(\varepsilon_{a b c d} R^{a b} \wedge e^{c} \wedge e^{d}-8 \bar{\psi} \wedge e_{a} \wedge \gamma^{a} \gamma_{5} \mathcal{D} \psi\right) \\
& +\frac{1}{4} f \wedge{ }^{*} f+\frac{\lambda}{4} \phi^{4 *} 1 .
\end{aligned}
$$


The equations of motion can be found by taking variations of Eq. (34) with respect to gauge fields $\omega^{a b}(x), e^{a}(x), \chi(x)$, $\bar{\psi}^{\alpha}(x)$ and $\phi(x)$, respectively,

$$
\begin{aligned}
0= & \varepsilon_{a b c d} \mathcal{D} e^{c} \wedge e^{d}+i \bar{\psi} \wedge e^{c} \wedge \gamma_{c} \sigma_{a b} \gamma_{5} \psi \\
0= & \frac{\phi^{2}}{\kappa}\left(\varepsilon_{a b c d} R^{b c} \wedge e^{d}+4 \bar{\psi} \wedge \gamma_{a} \gamma_{5} \mathcal{D} \psi\right) \\
& +\frac{1}{2}\left(f_{a b} e^{b} \wedge{ }^{*} f-\frac{1}{2} \varepsilon_{a b c d} f^{b c} e^{d} \wedge f\right)-\frac{\lambda \phi^{4}}{2}{ }^{*} e_{a} \\
0= & \frac{\phi^{2}}{\kappa} \bar{\psi} \wedge e_{a} \wedge \gamma^{a} \gamma_{5} \psi+\frac{1}{2} \mathcal{D}^{*} f \\
0= & e^{c} \wedge \gamma_{c} \gamma_{5} \mathcal{D} \psi \\
0= & \left(\varepsilon_{a b c d} R^{a b} \wedge e^{c} \wedge e^{d}-8 \bar{\psi} \wedge e_{a} \wedge \gamma^{a} \gamma_{5} \mathcal{D} \psi\right)-2 \kappa \lambda \phi^{2 *} 1
\end{aligned}
$$

Let us analyze Eq. (35) which is known as the torsion equation,

$$
\begin{aligned}
\varepsilon_{a b c d} \mathcal{D} e^{c} \wedge e^{d} & =-i \bar{\psi} \wedge e^{c} \gamma_{c} \sigma_{a b} \gamma_{5} \wedge \psi \\
& =-\frac{1}{2} \bar{\psi} \wedge \gamma_{5} e^{c} \gamma_{c}\left(\gamma_{a} \gamma_{b}-\gamma_{b} \gamma_{a}\right) \wedge \psi \\
& =i \epsilon_{a b c d} \bar{\psi} \gamma^{c} \wedge \psi \wedge e^{d}
\end{aligned}
$$

where we used the expressions $\gamma_{c} \gamma_{a} \gamma_{b}=\eta_{c a} \gamma_{b}-\eta_{c b} \gamma_{a}+$ $\eta_{a b} \gamma_{c}-i \epsilon_{c a b d} \gamma_{5} \gamma^{d}$ and $\bar{\psi} \gamma_{5} \gamma_{a} \psi=0$. If we compare the both sides of Eq. (40), we obtain $\mathcal{D} e^{c}=i\left(\bar{\psi} \gamma^{c} \wedge \psi\right)$ which satisfies the super-torsion constraint $\left(F^{a}=0\right)$. Moreover, using Eq. (36) and transforming the tangent indices to world space-time indices, one can show that the generalized Einstein field equations can be written as follows,

$R_{\alpha}^{\mu}-\frac{1}{2} \delta_{\alpha}^{\mu} R=T(\psi)_{\alpha}^{\mu}-\kappa \phi^{-2}\left\{T(\phi)_{\alpha}^{\mu}+\frac{1}{2} T(f)_{\alpha}^{\mu}\right\}$,

where,

$$
\begin{aligned}
T(\psi)_{\alpha}^{\mu} & =\bar{\psi}_{\nu} \gamma_{\alpha} \gamma_{5} \mathcal{D}_{[\rho} \psi_{\sigma]} \varepsilon^{\mu \nu \rho \sigma}, \\
T(\phi)_{\alpha}^{\mu} & =\frac{\lambda}{4} \delta_{\alpha}^{\mu} \phi^{4}, \\
T(f)_{\alpha}^{\mu} & =f_{\beta \alpha} f^{\mu \beta}+\frac{1}{4} \delta_{\alpha}^{\mu} f^{\gamma \delta} f_{\gamma \delta} .
\end{aligned}
$$

Here, as we mention before, we want to note that the additional spinorial field $\xi^{\alpha}(x)$ does not a new gravitino field because it contributes to neither the spacetime coordinate nor to the equations of motion. It only contributes to the boundary term.

Let us analyze the constraint $\mathcal{D} \phi=d \phi-\chi \phi=0$ which was proposed above to keep the scale invariance of the total action. This constraint gives a relation between the scalar compensating field and the dilatation gauge field as,

$\chi(x)=d \ln \phi(x)$.
Taking the exterior differential of the last equation, we find the dilatation curvature to be zero (i.e., $f=d \chi=0$ ). This is another way of expressing the inverse Higgs effect [51,52]. Clearly, this also shows the equivalence of the inverse Higgs constraint to equations of motion. From this constraint, the dilatation gauge field can be alternatively written as $\chi(x)=$ $d \alpha(x)$, where $\alpha(x)$ is a coordinate dependent field. Therefore, the scalar field can be described as a function of $\alpha(x)$,

$\phi(x)=e^{\alpha(x)}$

Since $f=0$, the expression $T(f)_{\alpha}^{\mu}$ goes to zero. As a result, Eq. (41) reduces to,

$R_{\alpha}^{\mu}-\frac{1}{2} \delta_{\alpha}^{\mu} R+\Lambda \delta_{\alpha}^{\mu}=T(\psi)_{\alpha}^{\mu}$,

where the cosmological term emerged as a function of the field $\alpha(x)$ as follows,

$\Lambda(x)=\frac{\kappa \lambda}{4} e^{2 \alpha(x)}$.

\section{Conclusion}

In this paper, we proposed an alternative way to obtain $D=4, \mathcal{N}=1$ supergravity lagrangian including scale transformations by using the Maxwell-Weyl superalgebra. This superalgebra contains two fermionic generators $Q_{\alpha}$ and $\Sigma_{\alpha}$, where the first one is the standard $\mathcal{N}=1$ fermionic generator and the other is required for the supersymmetrization of the Maxwell generator $Z_{a b}$ [21]. The new additional fermionic generators $\Sigma_{\alpha}$ were originally introduced by Green [12] in the context of superstring theory (see also [53]). Therefore, the Maxwell superalgebras can be considered as an extension of the Green algebra by adding the tensorial charges $Z_{a b}$.

We constructed the gauge theory of gravity based on $s \mathcal{M W}$ algebra. In this framework, we obtained the transformation of gauge fields, curvatures and the Bianchi identities of $s \mathcal{M W}$ algebra. The corresponding four-form lagrangian was constructed with bilinear of the curvatures together with a compensating scalar field. Our result can be considered as the Weyl enlargement of the paper [17] or/and supersymmetric generalization of the Maxwell-Weyl gravity [7]. According to Eq. (25), the Maxwell fields $B^{a b}(x)$, the new fermionic fields $\xi^{\alpha}(x)$ and the gauge field $A(x)$ appeared only in the boundary term. This result shows that the mentioned fields do not have a dynamical character, but the corresponding curvatures play an essential role for constructing an invariant action. We can say that analyzing the boundary term to find a physical or geometrical properties of the related fields is an open problem [54-56]. After analyzing the condition $\mathcal{D} \phi=$ 0 , we found the curvature of dilatation field goes to zero $f=$ $d \chi=0$. This condition also represents the Maurer-Cartan form of the dilatation field. Therefore, we have obtained an 
alternative way to express the inverse Higgs effect $[51,52]$. Moreover, we obtained a relation between the cosmological constant and the dilatation gauge field in Eq. (46).

We remark that the physical interpretation of the Maxwell symmetry and its supersymmetric extension are under research. According to the early studies [1,2], the Maxwell symmetry has been used to describe a particle moving in a Minkowski spacetime filled with a constant EM background field, parametrized by additional degrees of freedom related to the central charges $Z_{a b}$. So this symmetry was considered as the symmetry group of a particle moving in a constant EM field [57]. Later, the Maxwell algebra was used as an alternative way to generalize Einstein's theory of gravity and supergravity. In the gravitational framework, the additional degrees of freedom represent uniform gauge field strengths in (super)space which lead to uniform constant energy density [10]. By gauging the Maxwell (super)algebra, a generalized cosmological constant was proposed in $[4,18,19]$. In addition, the Maxwell gauge fields $B_{a b}(x)$ contributed to the stress energy-momentum tensor $T_{a b}(B)$ [4-7, 18, 19,34]. To our knowledge, this energy-momentum term has not been analyzed yet, but it is known that such an additional term may be related to the dark energy $[8,9]$. Also, the Maxwell symmetry provides a geometric background to define vector inflatons in cosmological models [58]. This symmetry was used to describe higher spin fields [25,26], planar dynamics of the Landau problem [27] and it was also applied to the string theory as an internal symmetry of the matter gauge fields [59]. Moreover, the cosmological consequences of the Maxwell symmetry are still an open problem. So, these results show the importance of the Maxwell symmetry.

Finally, considering the results in $[17-19,29,30]$ plus our findings, it is shown that the Maxwell superalgebras provide an effective geometric framework to construct supergravity lagrangians by using its curvatures.

\section{Notations and conventions}

Here, we summarize our notation and conventions in $D=4$. The Dirac gamma matrices are defined by the well-known expression $\left\{\gamma^{a}, \gamma^{b}\right\}=2 \eta^{a b}$ where $\eta_{a b}$ is the (mostly minus) Minkowski metric and these matrices obey the following relations,

$$
\begin{aligned}
\gamma^{5} & =\gamma_{5}=i \gamma^{0} \gamma^{1} \gamma^{2} \gamma^{3}, \quad\left(\gamma^{5}\right)^{2}=1, \quad\left(\gamma_{5}\right)^{\dagger}=\gamma_{5}, \\
\sigma^{a b} & =\frac{i}{2}\left[\gamma^{a}, \gamma^{b}\right], \quad \gamma_{5} \sigma_{a b}=\frac{i}{2} \varepsilon_{a b c d} \sigma^{c d}, \\
\left\{\gamma^{a}, \gamma^{5}\right\} & =0, \quad\left[\sigma^{a b}, \gamma^{5}\right]=0, \\
{\left[\sigma^{a b}, \gamma^{c}\right] } & =2 i\left(\eta^{[b \mid c} \gamma^{a]}\right), \quad\left\{\sigma^{a b}, \gamma^{c}\right\}=2 \varepsilon^{a b c d} \gamma_{5} \gamma_{d},
\end{aligned}
$$

$$
\begin{aligned}
{\left[\sigma^{a b}, \gamma^{5} \gamma^{c}\right] } & =2 i \gamma^{5}\left(\eta^{[b \mid c} \gamma^{a]}\right), \quad\left\{\sigma^{a b}, \gamma^{5} \gamma^{c}\right\}=2 \varepsilon^{a b c d} \gamma_{d} \\
{\left[\sigma^{a b}, \sigma^{c d}\right] } & =i\left(\eta^{a[d} \sigma^{b \mid c]}+\eta^{b[c} \sigma^{a \mid d]}\right) \\
\left\{\sigma^{a b}, \sigma^{c d}\right\} & =2\left(\eta^{a[c} \eta^{b \mid d]}+i \varepsilon^{a b c d} \gamma^{5}\right)
\end{aligned}
$$

where $\sigma^{a b}=-\sigma^{b a}$ are $O(3,1)$ Lorentz generators and the antisymmetrization is defined by $A^{[c} B^{d]}=A^{c} B^{d}-A^{d} B^{c}$. Moreover, we have,

$$
\begin{aligned}
\gamma_{a} \sigma^{a b} & =3 i \gamma^{b}, \quad \sigma^{a b} \sigma_{a b}=12, \\
\gamma_{c} \sigma^{a b} \gamma^{c} & =0, \quad \sigma_{a b} \gamma_{c} \sigma^{a b}=0, \\
\sigma_{a b} \sigma_{c d} \sigma^{a b} & =0, \quad \varepsilon_{a b c d} \sigma^{c d}=-2 i \gamma^{5} \sigma_{a b} .
\end{aligned}
$$

In this work, we use the Majorana spinors which satisfy $\bar{\psi}=\psi^{T} C$. Here, $C=\gamma_{0}$ represents the charge conjugation matrix and satisfies the following relations,

$$
\begin{aligned}
C^{T} & =C^{-1}=-C, \quad C^{2}=-1, \\
\left(C \gamma_{a}\right)^{T} & =\left(C \gamma_{a}\right), \quad\left(C \sigma_{a b}\right)^{T}=\left(C \sigma_{a b}\right), \\
\left(C \gamma_{5}\right)^{T} & =-\left(C \gamma_{5}\right), \quad\left(C \gamma_{a} \gamma_{5}\right)^{T}=-\left(C \gamma_{a} \gamma_{5}\right),
\end{aligned}
$$

where $T$ denotes the transpose of the corresponding matrix. For the closure of the superalgebras, the following cyclic identities are required,

$$
\left(C \gamma_{e}\right)_{(\alpha \beta}\left(C \gamma^{e}\right)_{\rho \sigma)}=0 \text {. }
$$

The spinor variables satisfy the following relations,

$$
\begin{aligned}
\psi_{\alpha} \chi_{\beta} & =-\chi_{\beta} \psi_{\alpha}, \quad \psi \chi \chi=\chi \psi, \\
\bar{\psi} \chi & =\bar{\chi} \psi, \quad \bar{\psi} \bar{\chi}=\bar{\chi} \bar{\psi}, \\
\bar{\psi} \gamma_{a} \chi & =-\bar{\chi} \gamma_{a} \psi, \quad \bar{\psi} \gamma_{a} \psi=0, \\
\bar{\psi} \sigma_{a b} \chi & =-\bar{\chi} \sigma_{a b} \psi, \quad \bar{\psi} \sigma_{a b} \psi=0, \\
\bar{\psi} \gamma_{5} \gamma_{a} \chi & =\bar{\chi} \gamma_{5} \gamma_{a} \psi, \quad \bar{\psi} \gamma_{5} \chi=\bar{\chi} \gamma_{5} \psi .
\end{aligned}
$$

If spinors have differential form structure, we have to consider the following relationships,

$$
\begin{aligned}
& \psi_{\alpha}^{(p)} \wedge \chi_{\beta}^{(q)}=-(-1)^{p \cdot q} \chi_{\beta} \wedge \psi_{\alpha}, \\
& \bar{\psi}^{(p)} \wedge \chi^{(q)}=(-1)^{p \cdot q} \bar{\chi} \wedge \psi .
\end{aligned}
$$

Considering a one-form spinor $\psi$, we have the following Fierz identities,

$$
\begin{aligned}
\psi \wedge \bar{\psi} & =\frac{1}{2} \gamma_{a} \bar{\psi} \wedge \gamma^{a} \psi-\frac{1}{8} \sigma_{a b} \bar{\psi} \wedge \sigma^{a b} \psi, \\
\gamma_{a} \psi \wedge \bar{\psi} \wedge \gamma^{a} \psi & =\sigma_{a b} \psi \wedge \bar{\psi} \wedge \sigma^{a b} \psi=0
\end{aligned}
$$

Acknowledgements The work of S.K. has been partly supported by the Scientific and Technological Research Council of Turkey (TUBITAK) under the grant number 2214-A. The authors also wish to thank J. Kowalski-Glikman for useful discussions and their kind hospitality at Institute for Theoretical Physics, University of Wrocław, where a part of this work was done. 
Data Availability Statement This manuscript has no associated data or the data will not be deposited. [Authors' comment: This is a theoretical work, hence no data is necessary.]

Open Access This article is distributed under the terms of the Creative Commons Attribution 4.0 International License (http://creativecomm ons.org/licenses/by/4.0/), which permits unrestricted use, distribution, and reproduction in any medium, provided you give appropriate credit to the original author(s) and the source, provide a link to the Creative Commons license, and indicate if changes were made.

Funded by SCOAP ${ }^{3}$.

\section{References}

1. H. Bacry, P. Combe, J.L. Richard, Nuovo Cimento 67, 267-299 (1970)

2. R. Schrader, Fortschr. Phys. 20, 701 (1972)

3. D.V. Soroka, V.A. Soroka, Phys. Lett. B 607, 302-305 (2005)

4. J.A. de Azcárraga, K. Kamimura, J. Lukierski, Phys. Rev. D 83, 124036 (2011)

5. D.V. Soroka, V.A. Soroka, Phys. Lett. B 707, 160-162 (2012)

6. R. Durka, J. Kowalski-Glikman, M. Szczachor, Mod. Phys. Lett. A 26, 2689 (2011)

7. O. Cebecioğlu, S. Kibaroğlu, Phys. Rev. D 90, 084053 (2014)

8. J. Frieman, M. Turner, D. Huterer, Ann. Rev. Astron. Astrophys. 46, 385 (2008)

9. T. Padmanabhan, Adv. Sci. Lett. 2, 174 (2009)

10. S. Bonanos, J. Gomis, K. Kamimura, J. Lukierski, Phys. Rev. Lett. 104, 090401 (2010)

11. R. D'Auria, P. Fré, Nucl. Phys. B 201, 101 (1982)

12. M.B. Green, Phys. Lett. B 223, 157-164 (1989)

13. J.A. de Azcárraga, J.M. Izquierdo, J. Lukierski, M. Woronowicz, Nucl. Phys. B 869, 303 (2013)

14. P.K. Concha, E.K. Rodríguez, Nucl. Phys. B 886, 1128 (2014)

15. J.A. de Azcárraga, J.M. Izquierdo, M. Picon, O. Varela, Class. Quantum Gravity 21, S1375 (2004)

16. F. Izaurieta, E. Rodríguez, P. Salgado, J. Math. Phys. 47, 123512 (2006)

17. J.A. de Azcárraga, J.M. Izquierdo, Nucl. Phys. B 885, 34-45 (2014)

18. P.K. Concha, E.K. Rodriguez, P. Salgado, JHEP 08, 009 (2015)

19. R. Durka, K. Kowalski-Glikman, M. Szczachor, Mod. Phys. Lett. A 27, 1250023 (2012)

20. J. Lukierski, Proc. Steklov Inst. Math. 272, 1-8 (2011)

21. K. Kamimura, J. Lukierski, Phys. Lett. B 707, 292-297 (2012)

22. J. Gomis, K. Kamimura, J. Lukierski, JHEP 08, 39 (2009)

23. S. Bonanos, J. Gomis, J. Phys. A Math. Theor. 42, 145206 (2009)

24. S. Bonanos, J. Gomis, K. Kamimura, J. Lukierski, J. Math. Phys. 51, $102301(2010)$

25. S. Fedoruk, J. Lukierski, JHEP 02, 128 (2013)
26. S. Fedoruk, J. Lukierski, J. Phys. Conf. Ser. 474, 012016 (2013)

27. S. Fedoruk, J. Lukierski, Phys. Lett. B 718, 646 (2012)

28. S. Hoseinzadeh, A. Rezaei-Aghdam, Phys. Rev. D 90, 084008 (2014)

29. P.K. Concha, E.K. Rodriguez, JHEP 09, 090 (2014)

30. P.K. Concha, O. Fierro, E.K. Rodriguez, P. Salgado, Phys. Lett. B 750, 117-121 (2015)

31. O. Cebecioğlu, S. Kibaroğlu, Phys. Lett. B 751, 131-134 (2015)

32. S. Kibaroğlu, O. Cebecioğlu, Mod. Phys. Lett. A 34, 1950016 (2019)

33. D.M. Peñafiel, L. Ravera, Fortschr. Phys. 65, 1700005 (2017)

34. D.M. Peñafiel, L. Ravera, Eur. Phys. J. C 78, 945 (2018)

35. L. Ravera, Eur. Phys. J. C 78, 211 (2018)

36. M. Omote, Lett. Nuovo Cimento 2, 58 (1971)

37. A. Bregman, Prog. Theor. Phys. 49, 667 (1973)

38. J.M. Charap, W. Tait, Proc. R. Soc. A 340, 249 (1974)

39. M. Kasuya, Nuovo Cimento Soc. Ital. Fis. 28B, 127 (1975)

40. O.V. Babourova, B.N. Frolov, VCh. Zhukovsky, Phys. Rev. D 74, $064012(2006)$

41. M. Blagojevic, Gravitation and Gauge Symmetries (IOP, Bristol, 2002)

42. H. Weyl, Space, Time, Matter (Dover, New York, 1961)

43. C. Brans, R.H. Dicke, Phys. Rev. 124, 925 (1961)

44. P.A.M. Dirac, Proc. R. Soc. A 333, 403 (1973)

45. T. Dereli, R.W. Tucker, Phys. Lett. 110B, 206 (1982)

46. A.G. Agnese, Phys. Rev. D 12, 3804 (1975)

47. E.S. Fradkin, A.A. Tseytlin, Phys. Rep. 119, 233-362 (1985)

48. M. Kaku, P.K. Townsend, P. van Nieuwenhuizen, Phys. Rev. D 17, 3179 (1978)

49. P. van Nieuwenhuizen, Int. J. Mod. Phys. A 1, 155-191 (1986)

50. P. van Nieuwenhuizen, Phys. Rep. 68, 189-398 (1981)

51. E.A. Ivanov, V.I. Ogievetsky, Teor. Mat. Fiz. 25, 164 (1975)

52. I. Low, A.V. Manohar, Phys. Rev. Lett. 88, 101602 (2002)

53. E. Bergshoeff, E. Sezgin, Phys. Lett. B 354, 256 (1995)

54. T. Regge, C. Teitelboim, Ann. Phys. 88, 286-318 (1974)

55. S.W. Hawking, G.T. Horowitz, Class. Quantum Grav. 13, 14871498 (1996)

56. D.A. Easson, P.H. Frampton, G.F. Smoot, Phys. Lett. B 696, $273-$ 277 (2011)

57. S. Bonanos, J. Gomis, J. Phys. A 43, 015201 (2010)

58. J.A. de Azcárraga, K. Kamimura, J. Lukierski, Int. J. Mod. Phys. Conf. Ser. 23, 01160 (2013)

59. S. Hoseinzadeh, A. Rezaei-Aghdam, Eur. Phys. J. C 75, 227 (2015) 